\title{
FURTHER RESULTS ON EXPANSIVE MAPPINGS
}

\author{
RICHARD K. WILLIAMS
}

\begin{abstract}
In this paper, several theorems are proved concerning the concepts of expansiveness and asymptoticity from topological dynamics. The results are derived using the techniques the author developed in a previous paper in this journal.
\end{abstract}

In [8], this author showed how the concept of an expansive homeomorphism could be generalized to that of an expansive continuous relation (called an expansive mapping), and that several of the well-known theorems on expansive homeomorphisms generalized in this new setting.

One reason expansive mappings are important is that they furnish the only routine technique (by using the shift transformation) for producing expansive homeomorphisms. One surprising result the technique has yielded is a Čechhomologically trivial continuum supporting an expansive homeomorphism. (See [8].)

It is useful to know how expansive mappings resemble expansive homeomorphisms so that one might gain some insight as to what surprises this technique might not produce. In this paper, several more well-known theorems will be generalized by using the tools developed in [8].

For reference purposes, the basic definitions and techniques from [8] will now be given.

If $X$ is a metric space with metric $d$, and if $f$ is a homeomorphism of $X$ onto itself, then $f$ is said to be expansive on $X$ with expansive constant $\delta>0$ if $x, y \in X, x \neq y$, implies $d\left(f^{n}(x), f^{n}(y)\right)>\delta$ for some integer $n$. Distinct points $x$ and $y$ are said to be positively (negatively) asymptotic under $f$ if for each $\varepsilon>0$, there is an integer $N$ such that $n>N(n<N)$ implies $d\left(f^{n}(x), f^{n}(y)\right)<\varepsilon$. Naturally, $x$ and $y$ are doubly asymptotic if they are both positively and negatively asymptotic.

Let $f$ be a continuous multivalued transformation of $X$ onto itself. ( $f$ is continuous at $x \in X$ if for each neighborhood $N$ of $f(x)$, there exists $\eta>0$ such that $d(x, y)<\eta$ implies $f(y) \subseteq N$.) Henceforth, a continuous multivalued transformation will simply be called a mapping.

Definition 1. Let $x \in X$. The orbit of $x$ under $f$ is defined by $O(x)$ $=\cup_{n=-\infty}^{\infty} f^{n}(x)$.

Definition 2. Let $x \in X$. A suborbit of $x$ under $f$ is a set of the form

Received by the editors October 18, 1975 and, in revised form, December 15, 1975.

AMS (MOS) subject classifications (1970). Primary 54H20; Secondary 54C60, 54C65.

Key words and phrases. Expansive function, expansive mapping, expansive homeomorphism, orbit, iterates, asymptotic points.

(1) American Mathematical Society 1976 
$\left\{x_{i}: x_{0}=x, x_{i+1} \in f\left(x_{i}\right)\right.$ for each integer $\left.i\right\}$.

DEFINITION 3. The mapping $f$ is expansive on $X$ with expansive constant $\delta>0$ if $x, y \in X, x \neq y$ implies for each suborbit $A$ of $x$ and for each suborbit $B$ of $y$, there exist $x_{n} \in A, y_{n} \in B$ such that $d\left(x_{n}, y_{n}\right)>\delta$.

Definition 4. Distinct points $x$ and $y$ are positively (negatively) asymptotic under $f$ if for each $\varepsilon>0$, there is an integer $N$ such that $n>N(n<N)$ implies $\inf \left\{d(a, b): a \in f^{n}(x), b \in f^{n}(y)\right\}<\varepsilon$.

It is clear that these definitions reduce to the standard ones when $f$ is a homeomorphism.

Let $X$ be bounded and let $f$ be a mapping of $X$ onto itself. Let $S(X)$ be the set of all single-valued transformations from the integers into $X$. For each $z \in S(X)$, denote $z(i)$ by $z_{i}$. Let $\varepsilon$ be a positive number. For $z, w \in S(X)$, define

$$
\rho(z, w)=d\left(z_{0}, w_{0}\right)+\varepsilon \sum_{i \neq 0} \frac{d\left(z_{i}, w_{i}\right)}{2^{|i|}} .
$$

Then it is readily verified that $\rho$ is a metric for $S(X)$. Call the resulting space $S(X, \varepsilon)$.

Let $S(X, f, \varepsilon)=\left\{z \in S(X, \varepsilon): z_{i+1} \in f\left(z_{i}\right)\right.$ for each $\left.i\right\}$, and let $h$ be the transformation of $S(X, \varepsilon)$ onto itself defined by $(h(z))_{i}=z_{i+1}$ for each $i$. (Thus $h$ is the "shift" transformation.)

The spaces $S(X, \varepsilon)$ and $S(X, f, \varepsilon)$ and the transformation $h$ have the following properties:

(1) The product and metric topologies for $S(X)$ are equivalent.

(2) The space $S(X, f, \varepsilon)$ is a closed subset of $S(X, \varepsilon)$; thus if $X$ is compact, so is $S(X, f, \varepsilon)$.

(3) The transformation $h$ is a homeomorphism of $S(X, f, \varepsilon)$ onto itself.

(4) The transformation $h$ is an expansive homeomorphism on $S(X, f, \varepsilon)$ if and only if $f$ is an expansive mapping on $X$.

(5) If $X$ is homeomorphic to $Y$ under $g$, then $S(X, f, \varepsilon)$ is homeomorphic to $S\left(Y, g f g^{-1}, \varepsilon\right)$ under the transformation $H$ defined by $(H(z))_{i}=g\left(z_{i}\right)$. Also, $h_{2}=H h_{1} H^{-1}$, where $h_{1}$ and $h_{2}$ are the shift transformations on $S(X, f, \varepsilon)$ and $S\left(Y, g f g^{-1}, \varepsilon\right)$, respectively.

(6) If $z, w \in S(X, f, \varepsilon)$, if $z_{i} \neq w_{i}$, and if $z$ and $w$ are positively (negatively) asymptotic under $h$, then $z_{i}$ and $w_{i}$ are positively (negatively) asymptotic under $f$.

These are the basic results needed to derive the previously mentioned generalizations.

Of the first six theorems, only Theorem 3 will be proven. The proofs of the other five are either similar to the proof of Theorem 3 or are routine modifications of the proofs of their counterparts in the literature. The last three theorems, which are the most important ones and whose proofs are the least trivial, will be proven completely.

THEOREM 1. Let $X$ have at least one limit point, and let $f$ be an expansive homeomorphism on $X$ with expansive constant $\delta$. Then $m \neq n$ implies $\sup \left\{d\left(f^{m}(x), f^{n}(x)\right): x \in X\right\}>\delta .($ See $[1$, Theorem 2].) 
THEOREM 2. Let $f$ be a single-valued uniformly continuous function which maps $X$ onto itself. Then $x$ and $y$ are positively (negatively) asymptotic under $f$ if and only if they are positively (negatively) asymptotic under $f^{n}$ for $n>0$. (See [3, Theorem 10.26].)

THEOREM 3. Let $X$ be compact, and let $f$ be a single-valued expansive mapping on $X$. If $p$ is a nonisolated periodic point under $f$, then there exists $x$ such that $p$ and $x$ are either positively or negatively asymptotic under $f$. (See [5, Theorem 2.4].)

Proof. It is shown in [6] that $f$ is expansive if and only if $f^{n}$ is expansive for $n \neq 0$. Thus we may assume that $p$ is fixed. Let $z \in S(X, f, 1)$ be defined by $z_{i}=p$ for each $i$. Let $\eta>0$ be given. Choose $N$ such that

$$
\sum_{|i|>N} \frac{\operatorname{diam}(X)}{2^{|i|}}<\frac{\eta}{2}
$$

Choose $q \neq p \in X$ such that $d\left(f^{i}(q), f^{i}(p)\right)<\eta / 6$ for $i=0,1, \ldots, 2 N$. There exists $w \in S(X, f, 1)$ such that $w_{-N}=q$. Then

$$
\begin{aligned}
\rho(z, w) & =\sum_{|i| \leqslant N} \frac{d\left(z_{i}, w_{i}\right)}{2^{|i|}}+\sum_{|i|>N} \frac{d\left(z_{i}, w_{i}\right)}{2^{|i|}} \\
& <\frac{\eta}{6} \sum_{|i| \leqslant N} \frac{1}{2^{|i|}}+\sum_{|i|>N} \frac{\operatorname{diam}(X)}{2^{|i|}} \\
& <\frac{\eta}{6} \cdot 3+\frac{\eta}{2}=\eta,
\end{aligned}
$$

and so $z$ is nonisolated in $S(X, f, 1)$. Also $z$ is fixed under $h$, so that there eixsts $v \in S(X, f, 1)$ such that $z$ and $v$ are either positively or negatively asymptotic under $h$, by Theorem 2.4 of [5]. By property 6 , there exists $x \in X$ such that $x$ and $p$ are either positively or negatively asymptotic under $f$. An application of Theorem 2 completes the proof.

THEOREM 4. If $X$ is compact and infinite, and if $f$ is a single-valued expansive mapping on $X$, there exists a point that is not periodic under $f$. (See the proof of Theorem 2 of [2].)

The following theorem generalizes Theorem 1 of this paper.

THeORem 5. Let $X$ have at least one limit point, and let $f$ be a single-valued expansive mapping on $X$ with expansive constant $\delta$. Let $m \geqslant 0, n \geqslant 0, m \neq n$. Then $\sup \left\{d\left(f^{m}(x), f^{n}(x)\right): x \in X\right\}>\delta$.

THEOREM 6. Let $X$ be compact, and let $f$ be a single-valued expansive mapping on $X$. Then $f$ has only a finite number of points of each period. (See [5, Theorem 3.1].)

THEOREM 7. Let $X$ be compact, and let $f$ be an expansive mapping on $X$ with expansive constant $\delta$. Then for each $\theta$ such that $0<\theta \leqslant \delta$, there exists a positive integer $k$ such that $d(x, y)>\theta$ implies that for each suborbit $A$ of $x$ and for each suborbit $B$ of $y$, there exists $n$ such that $|n| \leqslant k$ and $d\left(x_{n}, y_{n}\right)>\delta$. (See [1, Theorem 5].) 
Proof. By Theorem 3 of [8], the least upper bound of expansive constants for $f$ is not an expansive constant. Choose $\delta^{\prime}>\delta$ such that $\delta^{\prime}$ is an expansive constant for $f$, and choose a positive integer $i$ such that $2 M / i<\delta^{\prime}-\delta$, where $M=\operatorname{diam}(X)$. Let $0<\theta \leqslant \delta<\delta^{\prime}$. Using Theorem 5 of [1], select a positive integer $k$ such that $\rho(z, w)>\theta$ implies $\rho\left(h^{n}(z), h^{n}(w)\right)>\delta^{\prime}$ for some $n$ such that $|n| \leqslant k$. Let $d(x, y)>\theta$ and let $A$ be a suborbit of $x$ and let $B$ be a suborbit of $y$. Define $z, w \in S(X, f, 1 / i)$ in the natural way from the $x_{n}$ 's in $A$ and the $y_{n}$ 's in $B$, i.e., let $z_{n}=x_{n}$ and $w_{n}=y_{n}$ for each $n$. Now $\rho(z, w)$ $\geqslant d\left(z_{0}, w_{0}\right)=d(x, y)>\theta$, so

$$
\rho\left(h^{n}(z), h^{n}(w)\right)=d\left(z_{n}, w_{n}\right)+\frac{1}{i} \sum_{j \neq 0} \frac{d\left(z_{n+j}, w_{n+j}\right)}{2^{|j|}}>\delta^{\prime},
$$

where $|n| \leqslant k$. Hence, $d\left(z_{n}, w_{n}\right)>\delta$, i.e., $d\left(x_{n}, y_{n}\right)>\delta$. Q.E.D.

The following theorem is obviously related to Theorem 1 of this paper.

THEOREM 8. Let $X$ have at least one limit point, and let $f$ be an expansive mapping on $X$. Let $\theta$ be the least upper bound of the expansive constants for $f$. Then $m \neq n$ implies

$$
\sup \left\{\sup \left\{d(a, b): a \in f^{m}(x), b \in f^{n}(x)\right\}: x \in X\right\} \geqslant \theta .
$$

Proof. Let $0<\alpha<\beta<\theta$. Then $\alpha$ and $\beta$ are expansive constants for $f$. Choose a positive integer $i$ such that $2 M / i<\beta-\alpha$, where $M=\operatorname{diam}(X)$. Since $\beta$ is an expansive constant for $f$, it is an expansive constant for $h$ on $S(X, f, 1 / i)$. Clearly $S(X, f, 1 / i)$ has at least one limit point if $X$ does, so by Theorem 1 , there exists $z \in S(X, f, 1 / i)$ such that $\rho\left(h^{m}(z), h^{n}(z)\right)>\beta$, i.e.,

$$
d\left(z_{m}, z_{n}\right)+\frac{1}{i} \sum_{j \neq 0} \frac{d\left(z_{m+j}, z_{n+j}\right)}{2^{|j|}}>\beta .
$$

Hence, $d\left(z_{m}, z_{n}\right)>\alpha$, and since $z_{m} \in f^{m}\left(z_{0}\right), z_{n} \in f^{n}\left(z_{0}\right)$,

$$
\sup \left\{\sup \left\{d(a, b): a \in f^{m}(x), b \in f^{n}(x)\right\}: x \in X\right\}>\alpha .
$$

But this is true for any $\alpha<\theta$. Hence,

$$
\sup \left\{\sup \left\{d(a, b): a \in f^{m}(x), b \in f^{n}(x)\right\}: x \in X\right\} \geqslant \theta .
$$

COROLlARY. If $X$ is compact and infinite, and if $f$ is an expansive mapping on $X$ with expansive constant $\delta$, then $m \neq n$ implies

$$
\sup \left\{\sup \left\{d(a, b): a \in f^{m}(x), b \in f^{n}(x)\right\}: x \in X\right\}>\delta .
$$

(See [1, Theorem 2].)

Proof. By Theorem 3 of [8], the least upper bound of the expansive constants is not an expansive constant. The result now follows from Theorem 8.

A different proof of the preceding corollary can be found in [8, p. 658].

THEOREM 9. Let $X$ be compact and let $f$ be a single-valued mapping of $X$ onto 
itself which is expansive on $X-A$, where $A$ is a finite subset of $X$. Then $f$ is expansive on $X$. (See [1, Theorem 3].)

Proof. Let $\delta$ be an expansive constant for $f$ on $X-A$. Let $A$ consist of nonperiodic points $x_{1}, x_{2}, \ldots, x_{n}$, and periodic points $y_{1}, y_{2}, \ldots, y_{m}$. Let $y_{i}$ be of period $p_{i}$. Define $z^{i} \in S(X, f, \varepsilon)$ by $z_{j p_{i}}^{i}=y_{i}, j=0, \pm 1, \ldots$ (This defines $z^{i}$, since $f$ is single-valued.) Let $z, w \in S(X, f, \varepsilon)-\cup_{i=1}^{m} O\left(z^{i}\right)$ with $z \neq w$. Let $1 \leqslant i \leqslant m$, and let $0 \leqslant j<p_{i}$. Since $z \notin O\left(z^{i}\right)$, there is an integer $k_{i j}$ such that $z_{k_{i j} p_{i}+j} \neq y_{i}$, and so $k<k_{i j}$ implies $z_{k p_{i}+j} \neq y_{i}$. Let $N=\min \left\{k_{i j} p_{i}+j\right\}$. We now show that there exists $N_{1}$ such that $k<N_{1}$ implies $z_{k} \notin A$. Let $0 \leqslant i \leqslant m$, and let $k<N$. Choose integers $r$ and $j$ such that $k=r p_{i}+j$, where $0 \leqslant j<p_{i}$. Since $k<N, r p_{i}+j<N \leqslant k_{i j} p_{i}+j$, so that $r<k_{i j}$. Hence, $z_{r p_{i}+j} \neq y_{i}$, i.e., $z_{k} \neq y_{i}$. Also, each $x_{i}$ appears as a coordinate of $z$ at most once, since $x_{i}$ is nonperiodic. Hence, there exists $N_{1}$ such that $k<N_{1}$, implies $z_{k} \notin A$. Also, there exists $N_{2}$ such that $k<N_{2}$ implies $w_{k} \notin A$. Since $z \neq w$, there exists $N_{3}$ such that $k<N_{3}$ implies $z_{k} \neq w_{k}$. Finally, if $M=\min \left\{N_{1}, N_{2}, N_{3}\right\}, k<M$ implies $z_{k} \neq w_{k}, z_{k} \notin A$, $w_{k} \notin A$. Choose $k<M$. Since the components of $z$ form a suborbit of $z_{k}$ and the components of $w$ form a suborbit of $w_{k}$, there is an integer $a$ such that $d\left(z_{k+a}, w_{k+a}\right)>\delta$, so, $\rho\left(h^{k+a}(z), h^{k+a}(w)\right) \geqslant d\left(z_{k+a}, w_{k+a}\right)>\delta$, and $h$ is expansive on $S(X, f, \varepsilon)-\bigcup_{i=1}^{m} O\left(z_{i}\right)$. By the theorem in [7], $h$ is expansive on $S(X, f, \varepsilon)$, so by property $4, f$ is expansive on $X$.

If $A$ contains no periodic points, then $h$ is expansive on $S(X, f, \varepsilon)$. For let $A=\left\{x_{1}, x_{2}, \ldots x_{n}\right\}$, where each $x_{i}$ is nonperiodic. Let $z, w \in S(X, f, \varepsilon)$ with $z \neq w$. As before, there is an integer $N$ such that $k<N$ implies $z_{k} \notin A, w_{k}$ $\notin A, z_{k} \neq w_{k}$. Let $k<N$. As above, there is an integer $a$ such that $\rho\left(h^{a}(z), h^{a}(w)\right)>\delta$, so that $h$ is expansive on $S(X, f, \varepsilon)$, and hence, $f$ is expansive on $X$.

\section{REFERENCES}

1. B. F. Bryant, Expansive self-homeomorphisms of a compact metric space, Amer. Math. Monthly 69 (1962), 386-391.

2. —, On expansive homeomorphisms, Pacific J. Math. 10 (1960), 1163-1167. MR 22 \#11382.

3. W. H. Gottschalk and G. A. Hedlund, Topological dynamics, Amer. Math. Soc. Colloq. Publ., vol. 36, Amer. Math. Soc., Providence, R.I., 1955. MR 17, 650.

4. William Reddy, The existence of expansive homeomorphisms on manifolds, Duke Math. J. 32 (1965), 627-632. MR 32 \#4679.

5. W. R. Utz, Unstable homeomorphisms, Proc. Amer. Math. Soc. 1 (1950), 769-774.

6. Richard K. Williams, A note on expansive mappings, Proc. Amer. Math. Soc. 22 (1969), 145-147. MR 39 \#3477.

7. - On expansive homeomorphisms, Amer. Math. Monthly 76 (1969), 176-178. MR 38 \#6554.

8. Some results on expansive mappings, Proc. Amer. Math. Soc. 26 (1970), 655-663. MR 42 \# 1094.

Department of Mathematics, Southern Methodist University, Dallas, TeXas 75275 